\title{
Study on the Influence of Metallic Powder in Near-Dry Electric Discharge Machining
}

\author{
Sanjay Sundriyal ${ }^{1}$ - Vipin ${ }^{1}$ - Ravinderjit Singh Walia2,* \\ 1Delhi Technological University, India \\ 2Punjab Engineering College, India
}

Near-dry electrical discharge machining (ND-EDM) is an eco-friendly process. In this study, an approach has been made to make the machining process more efficient than ND-EDM with the addition of metallic powder with the dielectric medium to machine EN-31 die steel. Powdermixed near-dry EDM (PMND-EDM) has several advantages over the ND-EDM or conventional electrical discharge machining (EDM) process, such as a higher material removal rate (MRR), fine surface finish (Ra), sharp cutting edge, lesser recast layer, and lower deposition of debris. The output response variables are MRR, Ra, residual stress (RS) and micro-hardness (MH) of the machined surfaces. Further study of the workpiece was performed, and a comparative study was conducted between ND-EDM and PMND-EDM. In this proposed method of machining, the MRR, Ra, and MH increased by $17.85 \%, 16.36 \%$, and $62.69 \%$ while RS was reduced by $56.09 \%$.

Keywords: material removal rate; near-dry EDM; powder mixed near-dry EDM; residual stress; surface roughness

\section{Highlights}

- Experimental investigations have been conducted in the PMND-EDM process. The metallic powder (zinc) as an additive along with the mist of a dielectric medium was used for performance enhancements.

- In PMND-EDM, the MRR was influenced by powder concentration. The MRR increases with the increase in metallic powder concentration, and the maximum increase in MRR was $17.85 \%$, as compared to ND-EDM.

- $\quad$ The surface finish was improved by $16.36 \%$ in PMND-EDM as the sparking is uniformly distributed among the powder particles, which gives even and uniform machined surfaces.

- $\quad$ There was a decrease in residual stress by $56.09 \%$ over the machined surfaces in PMND-EDM as compared to ND-EDM process.

- $\quad$ The maximum increase in MH was $62.69 \%$ over the machined surface in PMND-EDM as compared to ND-EDM.

\section{INTRODUCTION}

The necessity of machines using the least harmful cutting fluids has prompted many researchers to investigate the use of minimum quantity lubrication (MQL) in near-dry machining (NDM). In NDM, machining is done with the supply of minute quantities of lubricant or mist at the work surface. It was developed as an alternative technique to supply the internal high-pressure coolant, reducing the use of metal-working fluids (MWFs), which eventually also led to a reduction in the cost of MWFs. In NDM, the cooling medium is a combination of air and oil in the form of an aerosol, or minute droplets of mist. Mist is the gaseous suspension of solid into air (or liquid) particles. The process feasibility of near-dry electrical discharge machining (EDM) was explored in 1981, and further investigations were performed on dielectric mixtures of mist with gases such as argon and nitrogen [1]. In powder-mixed EDM (PM-EDM), the effect on the breakdown voltage of kerosene by the addition of graphite powder was studied [2]. It has been noted that some powders, such as graphite and silicon when mixed with dielectric, the distribution of spark at the discharge gap led to the creation of very fine glossy surfaces [3]. The MRR increases and tool wear rate (TWR) decreases with the proper addition of metallic powders to the dielectric fluid. An approach was made to achieve optimal process parameters setting in PM-EDM, and the study revealed that process parameters at optimum levels in PM-EDM gave higher MRR and surface finish [4]. The effect of different powder concentration of graphite powder on responses such as Ra, TWR and MRR on H-11 die steel was studied, revealing that the addition of graphite powder at a concentration of $6 \mathrm{~g} / \mathrm{l}$ enhances the MRR and surface finish while tool wear rate is decreased [5]. Research was conducted with different dielectrics on environmental and hazard and operability (HAZOP) analysis in PMEDM, concluding that HAZOP analysis successfully reduced the wastage of dielectric, and minimized the machining cost and environmental hazards as compared to traditional EDM machining methods [6]. The thermal phenomenon in powder-mixed near-dry electrical discharge machining (PMND-EDM) was explained, and the tendency of variation in MRR was analysed by varying each process parameter [7] to [9]. The plasma channel characteristics in PM-EDM were analysed [10]. It was proved that the plasma channel 
was much more stable in PM-EDM than the plasma formed in pure kerosene oil because the plasma generated in PM-EDM was compressed by the electric bridge of conductive powder particles in EDM oil. The machinability of titanium alloy (Ti-6Al-4V) was examined during EDM. The performance was assessed in terms of MRR, as was the rate of tool wear. The surface integrity of the machined specimen is evaluated in the purview of surface morphology and topographical features [11]. The impact of $\mathrm{SiC}$ powder concentration on surface topography, particle deposition, and subsurface structures in the PMEDM of Ti-6Al-4V-ELI work material was studied, and it was observed that a high suspended particle concentration in dielectric liquid enhance the material transfer mechanism in a particulate form [12]. Flushing mode plays an important role in any EDM operation. An incorrect flushing can result in erratic cutting and poor machining conditions [13]. Significant work has also been done in the field of residual stress generated over a machined surfaced by EDM, and an attempt has been made to model these stresses so that these residual stresses are minimized [14]. It was revealed that the value of residual stresses at the sub-surface of the workpiece machined by EDM was more than the top surface due to high surface roughness over the top surface. A parametric study for residual stresses was performed in wire EDM, utilizing the Taguchi method of optimization to minimize the residual stresses in aluminium workpieces [15]. A nano-indentation technique and Raman spectroscopy were adopted to measure the residual stress in AISI H13 tool steel machined by EDM process [16]. The optimization of the residual stress was done using an X-ray $\cos \alpha$ method in a vibration-assisted hybrid EDM process, analysing the residual stress generated over a high-carbon high-chromium D2 tool steel workpiece [17]. Several studies have been done regarding enhancing the micro-hardness $(\mathrm{MH})$ value and surface roughness of the machined components by powder-additive EDM methodology. Efforts have been made to identify favourable conditions to enhance the surface $\mathrm{MH}$ of EN-31 tool steel using $\mathrm{Cu}-\mathrm{W}$ tool manufactured by powder metallurgy in the electrical discharge alloying (EDA) process. The Taguchi method was followed to obtain a combination of process variables for achieving the best $\mathrm{MH}$. The presence of hard tungsten carbide $\left(\mathrm{W}_{2} \mathrm{C}\right)$ and cementite $\left(\mathrm{Fe}_{3} \mathrm{C}\right)$ on the machined surface was related to the observed substantial increase in $\mathrm{MH}$ (approx. $150 \%$ [18]. An experimental work was carried out for the surface modification of Ti6Al4V alloy using $\mathrm{TiC} / \mathrm{Cu}$ powder metallurgy electrode. Taguchi's $\mathrm{L}_{18}$ mixed orthogonal array and analysis of variance were performed to evaluate the influence of parameters on surface roughness and $\mathrm{MH}$. The carbides generated on the machined surface, increased the hardness as high as $912 \mathrm{HV}$ without much sacrifice of the roughness of the machined surface [19]. The surfaces of the die steel materials were modified by the addition of tungsten powder in a dielectric medium in EDM process [20]. This addition caused the deposition of carbon in the plasma, which improved the surface morphology and increased $\mathrm{MH}$ by more than $100 \%$. The $\mathrm{MH}$ of cryogenically treated aluminium alloy was improved by $94.85 \%$ in PM-EDM [21]. A Taguchi $\mathrm{L}_{9}$ orthogonal array was utilized to increase the $\mathrm{MH}$ of the workpiece in PMND-EDM and resulted in an increase of MHupto $506.63 \mathrm{HV}$ [22].

The use of minimum quantity lubrication system plays an important role regarding the accuracy obtained in machined products and also prevents environmental pollution and hazard to the workers. The use of optimum metal-working fluids leads to economic benefits and environmentally friendly machining methods. Although research related to PMND-EDM has been done previously, it was limited to the study of the $\mathrm{MH}$ of the machined component. This study aims to further investigate PMND-EDM in a detailed manner. After an extensive literature review, an attempt has been made to study PMNDEDM related to MRR, Ra, residual stress (RS) and $\mathrm{MH}$. In this research, a new setup was developed to study the role of three-phase dielectric with different electrode dimensions, metallic powder concentration, and flow rate. His experimental setup was developed to investigate the effects of metallic powder concentration, various electrodes, and dielectric flow rate on the machining performance on the abovementioned factors. Finally, the comparisons on machining performances were made between NDEDM and PMND-EDM based on the results obtained by experiments.

\section{METHODS}

\subsection{Indigenous Developed Setup for Powder Mixed Near- Dry EDM}

In modern manufacturing techniques, researchers are always developing highly productive machining methods. In comparison to conventional EDM, a recent trend in technology and innovation has brought in hybrid EDM, such as dry EDM, near-dry EDM and PMND-EDM. PMND-EDM machining gives a minimum heat-affected zone and a very low 
recast layer in the machined products. As discussed, PMND-EDM has several advantages over traditional EDM process; therefore, there is a need to develop a more efficient hybrid setup for PMND-EDM (Fig. 1). It has a minimal input of resources, is relatively environmentally friendly and also gives the desired results. In this study, an indigenous setup for PMNDEDM was developed that uses metallic powder mixed with kerosene oil (LL-221) under high air pressure as a dielectric medium. This machining process has advantages in terms of MRR, surface finish, and other surface characteristics in comparison to neardry EDM, dry EDM, and other conventional EDM methods. The dielectric in this indigenous setup is a heterogeneous mixture of three phases (solid + liquid + gas). A mixing chamber was designed and manufactured in which oil along with metallic powder and glycerol are mixed in right proportion with compressed air supplied from the compressor at a high pressure, ranging from $0.4 \mathrm{MPa}$ to $0.8 \mathrm{MPa}$. The setup was designed and developed in the Precision Manufacturing Laboratory (Delhi Technological University (DTU), Delhi). The designed chamber has a separate inlet for dielectric medium and, in case the pressure inside the chamber goes beyond operational limits, a safety valve mounted on top to release the pressure of compressed air has been provided. A flow meter was integrated with the setup, which can vary the flow rate of dielectric mist within range of $1 \mathrm{ml} /$ $\min$ to $20 \mathrm{ml} / \mathrm{min}$. As the flow meter is a transparent body, the flow rate of mist can be easily read from the calibration scale mounted on the flow meter. To avoid metallic powder particle settling, glycerol is added in the tank along with dielectric fluid from the inlet of the tank. The setup also includes manually operated regulators mounted on the control panel for regulating air and oil pressure ( $0 \mathrm{MPa}$ to $0.8 \mathrm{MPa})$. The setup

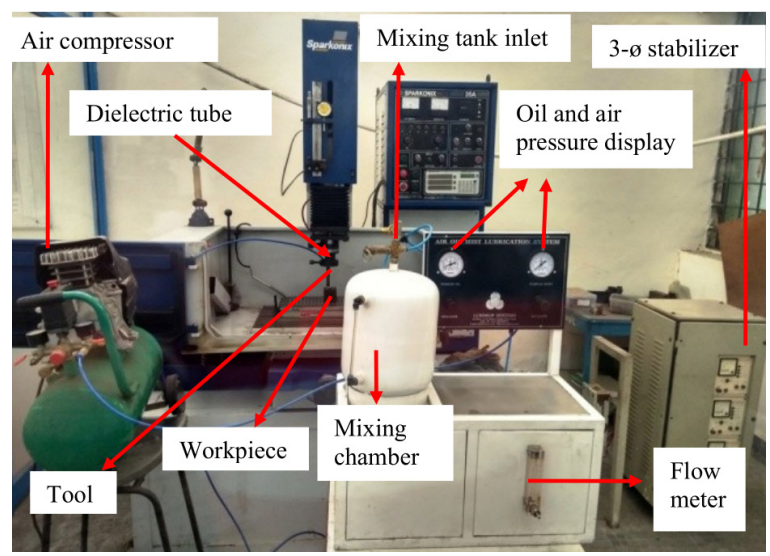

Fig. 1. Developed experimental setup for PMND-EDM comprises a display unit for oil and air pressure, which is analogous in nature. The control panel of the EDM machine (Sparkonix Limited, Pune), consists of electrical and non-electrical parameters, such as pulse on, pulse off, gap voltage, gap current, lift, sensitivity, feed and speed. Experimental conditions for machining and is shown in Table 1.

Table 1. Experimental condition for machining

\begin{tabular}{lc}
\hline Parameters & Classification \\
\hline Workpiece & EN-31 $(35 \mathrm{~mm} \times 15 \mathrm{~mm} \times 15 \mathrm{~mm})$ \\
\hline Tool electrode & COPPER \\
\hline Dielectric & Air + oil $(\mathrm{LL}-221)+$ metallic powder \\
\hline Discharge current & $12 \mathrm{~A}$ \\
\hline Gap voltage & $25 \mathrm{~V}$ \\
\hline Pulse on / off & $500 \mu \mathrm{s} / 75 \mu \mathrm{s}$ \\
\hline Polarity & $0 \mathrm{ve}$ \\
\hline Powder concentration $2 \mathrm{~g} / \mathrm{l}, 4 \mathrm{~g} / \mathrm{l}$ \\
\hline Flow meter of dielectric & $0 \mathrm{ml} / \mathrm{min}$ to $20 \mathrm{ml} / \mathrm{min}$ \\
\hline Air and oil pressure & $0.6 \mathrm{MPa}$ \\
\hline Powder material & Zinc $(22 \mu \mathrm{m})$ \\
\hline Stabilizing agent & Glycerol $(5 \%)$ \\
\hline
\end{tabular}

Fig. 2 shows the internal design of the tool. Different dimensions of the sparking end of the tools and properties of the workpiece are given in Tables 2 and 3, respectively. Hollow tubular copper electrodes, shown in Fig. 3, were developed in the precision manufacturing lab., DTU, Delhi.

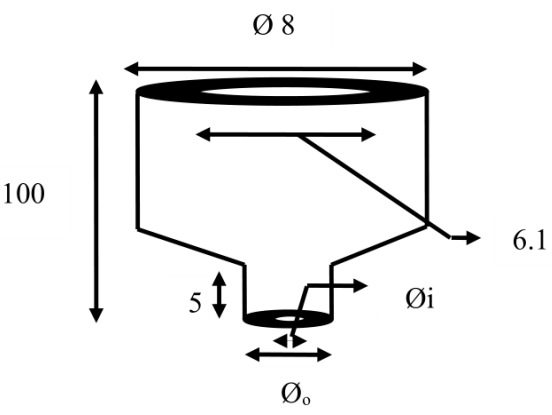

Fig. 2. Design of tool developed for PMND-EDM; all dimensions in $\mathrm{mm}$

Table 2. Diameter of various tools developed for present study (sparking end)

\begin{tabular}{ccc}
\hline Tool type & Inner diameter $[\mathrm{mm}]$ & Outer diameter $[\mathrm{mm}]$ \\
\hline Tool 1 & 1 & 2 \\
\hline Tool 2 & 2 & 3 \\
\hline Tool 3 & 3 & 4 \\
\hline Tool 4 & 4 & 5 \\
\hline
\end{tabular}


The other ends of the tools are constant in dimension with $6.1 \mathrm{~mm}$ inner diameter and $8 \mathrm{~mm}$ outer diameter. The tool setup was developed for PMND-EDM, which has a flexible tube of $6 \mathrm{~mm}$ outer diameter inserted at the top end of the tool.

$\varnothing 4$

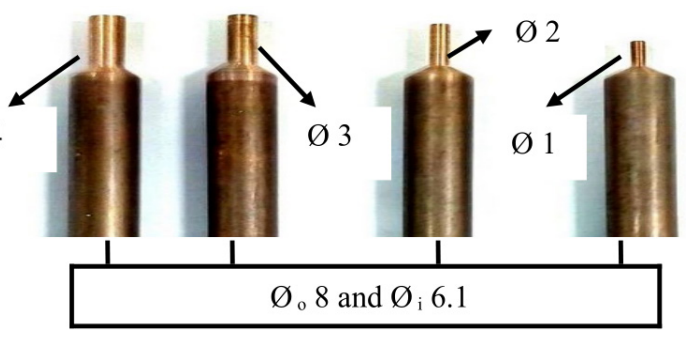

Fig. 3. Hollow copper electrode of different dimensions; all dimensions in $\mathrm{mm}$

Table 3. Properties of workpiece (EN-31)

\begin{tabular}{lc}
\hline Thermal conductivity $[\mathrm{W} /(\mathrm{m} \mathrm{K})]$ & 44.5 \\
\hline Hardness [HRC] & 63 \\
\hline Yield stress [MPa] & 450 \\
\hline Tensile strength [MPa] & 750 \\
\hline Density $\left[\mathrm{kg} / \mathrm{m}^{3}\right]$ & 7850 \\
\hline Melting point $\left[{ }^{\circ} \mathrm{C}\right]$ & 1540 \\
\hline
\end{tabular}

\section{EXPERIMENTAL}

\subsection{Working Principle of PMND-EDM}

The dielectric medium, which is a mixture of oil, metallic powder, comes out from the outlet of the mixing chamber in the form of mist accompanied by pressurized air, which was passed by the compressor into the mixing chamber. This mist was supplied to the electrode tool through a flexible tube, which eventually passes through the hollow passage of the copper tool, as shown in Fig. 4. The mist flow rate was controlled manually by the regulator provided at the control panel of the setup. As the desired experimentation condition was achieved, straight polarity power was supplied to the tool set up and the workpiece. The material removal rate is based on the heat erosion principle. The moment sparking is generated at the inter-electrode gap (IEG); gasification and fusion take place at the machining gap due to which the plasma channel is formed between the tool and the workpiece, providing a heat source to the surface of the workpiece [1]. The enormous amount of heat developed at the machining gap or spark gap melts the surface of workpiece and creates numerous craters, due to which erosion starts at the surface.
The eroded material or debris was flushed out by the high-pressurize mist. Due to the presence of metallic powder (additive), it was observed that a phenomenon of a chain like formation of powder particles, also known as the bridging effect which occurs at the machining gap [4].

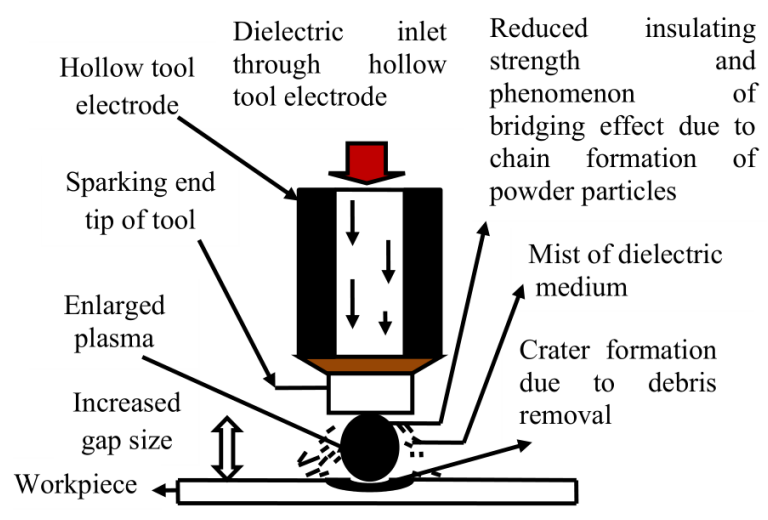

Fig. 4. Schematic diagram of electrode, plasma and workpiece interaction with each other

The chain-like formation of powder particles was due to the interlocking of grains when they come close together due to which the discharge gap between the tool and workpiece increases, energizing the spark plasma and leading to enlarged widened plasma channels with reduced electrical density. This bridging effect of grains decreases the dielectric insulating strength and gap voltage at the IEG. These favourable conditions show an increase of discharging frequency, further resulting in faster sparking at the workpiece surface due to which the material removal rate increases with improved surface quality.

\section{EXPERIMENTAL METHOD}

\subsection{Experimental Process Parameters}

The process parameters can be divided into different categories, i.e., electrical parameters, non-electrical parameters (other parameters), electrode parameters (tool), workpiece parameters and metallic powder parameters. The output response parameters include PMND-EDM process performance, such as MRR, $\mathrm{Ra}, \mathrm{RS}$ and $\mathrm{MH}$. The powder (zinc) used was metallic along with a stabilizing agent (glycerol) so that the powder uniformly mixes with the dielectric medium. The electrical parameters include current, pulse time, voltage, gap voltage, sensitivity, lift, feed and speed. Other parameters include mist pressure, flow rate, 
etc.; the remaining factors include the type of tool electrode and workpiece.

Electrical parameters:

- Peak current: This is the most important electrical parameter, which determines the spark energy.

- Discharge voltage: This is also known as gap voltage, which is responsible for the spark gap.

- Sensitivity: This regulator is used to control the movement of the tool.

- Speed: These control the speed of z-axis lead screw or the tool.

Non-electrical parameters:

- Oil and air pressure: These are regulated constantly to achieve a perfect phase of both in mist.

- Dielectric flushing pressure: This is the pressure of the mist coming out from the dielectric tank.

- Mist flow rate controller: This device controls the flow rate of dielectric mist, measured in $\mathrm{ml} / \mathrm{min}$.

- Workpiece and electrode tool: The workpiece selected for the study is EN-31, which has good physical and chemical properties, while the electrode tool selected was copper, due to good thermal conductivity and high boiling point.

- Dielectric oil: Kerosene based oil (LL-221) was chosen as it has good cooling and flushing ability.

- Metallic powder: Different metallic powders serve as additives in the dielectric medium such as copper, zinc, silicon, graphite, aluminium powder, etc.

\subsection{Experimental Procedure}

Series of experiments were conducted in this developed hybrid setup, and responses, such as MRR, $\mathrm{Ra}, \mathrm{RS}$, and $\mathrm{MH}$, were studied. The dielectric mixture was prepared by adding kerosene oil, zinc powder and glycerol. Once the mixture is filled in the dielectric tank, the pressure of mist was set at a value of 0.6 MPa by the regulator at the control panel of the hybrid setup. The flowmeter for the mist was set up at $6 \mathrm{ml} /$ min. The machining time of 10 minutes was set for all the experiments. The first set of experiments was performed with mist only (i.e., without any metallic powder); the second set of experiments was performed with $2 \%(2 \mathrm{~g} / \mathrm{l})$ powder concentration; the third set of experiments was done with $4 \%(4 \mathrm{~g} / \mathrm{l})$ powder concentration with four different tools as given in Table 4.

A total of 36 experiments (repeatability with three times for each experiment) were conducted under experimental settings (Table 5) for obtaining the average values for output responses. The experiments were conducted with positive polarity to obtain better results.

Table 4. Set for experiments

\begin{tabular}{cccc}
\hline Exp. no. & Powder concentration [\%] & Total experiments & Tool type \\
\hline 1 & 0 & 4 & $1,2,3,4$ \\
\hline 2 & 2 & 4 & $1,2,3,4$ \\
\hline 3 & 4 & 4 & $1,2,3,4$ \\
\hline Pressure 0.6 MPa; time $10 \mathrm{~min} ; T_{\text {on }} 500 \mu \mathrm{s} ; T_{\text {off }} 75 \mu \mathrm{s} ;$ \\
discharge current 12 A; gap voltage $25 \mathrm{~V}$; tool electrode (copper); \\
workpiece EN-31; mist flow rate $6 \mathrm{ml} / \mathrm{min}$
\end{tabular}

Table 5. Dielectric medium proportion for different set of experiments

\begin{tabular}{cc}
\hline Exp. no. & Medium \\
\hline 1 & Compressed Air + kerosene oil \\
\hline 2 & $\begin{array}{c}\text { Compressed Air }+2 \text { gram zinc powder }+ \\
\text { kerosene oil }+5 \% \text { glycerol }\end{array}$ \\
\hline 3 & $\begin{array}{c}\text { Compressed Air }+4 \text { gram zinc powder }+ \\
\text { kerosene oil }+5 \% \text { glycerol }\end{array}$ \\
\hline
\end{tabular}

The response was measured using the relations given below:

$$
M R R=\left(W_{i}-W_{f}\right) / T_{m},
$$

where $W_{i}$ is the initial weight of the workpiece before machining, $W_{f}$ the final weight of the workpiece after machining, $T_{m}$ the time taken for machining, and $M R R$ in $\mathrm{mg} / \mathrm{min}$.

Surface finish: Ra was checked by using a benchtop ZeGage optical profilometer (model TM3000).

Residual stress: RS was calculated by using a Pulsetech $\mu \mathrm{X}-360$ residual stress analyser.

Micro-hardness: MH was measured by using a Fischerscope instrument (HM2000S model, USA)

Weight of the specimen was measured using an electronic balance of least count 0.001gram (Asia Techno weigh India).

Scanning electron microscope: A Hitachi Scanning electron microscope for the study of surface morphology of machines surface.

\section{RESULTS AND DISCUSSION}

\subsection{Influence of Machining Process on MRR}

In PMND-EDM, with the addition of liquid and conductive metallic powder to compressed air, there was a reduction of the dielectric medium insulating strength between a tool and workpiece electrodes. This resulted in improved de-ionization effect and 
discharging conditions. All these factors improve discharge frequency and MRR is thus increased. A solid and liquid mixture, such as mist assisted with metallic powder, changes the electric field strength of the dielectric medium and thus facilitates the discharge initiation [7]. Enlarged discharge gap results in sufficient heat dissipation, due to which more molten materials were ejected by the explosive force by the gasification of solid and liquid phases. This phenomena also restrains the excessive expansion of the discharge channel in its radius. Higher MRR was observed in metallic PMND-EDM, because IEG increases due to the cluster formation of metallic powder, which increases plasma intensity and results in more erosion. The samples of the workpiece machined by PMND-EDM are shown in Fig. 5. It was experimentally proved that the maximum percentage increase in MRR of PMND-EDM was $17.85 \%$ as compared to near- dry EDM, as shown in Fig. 6.

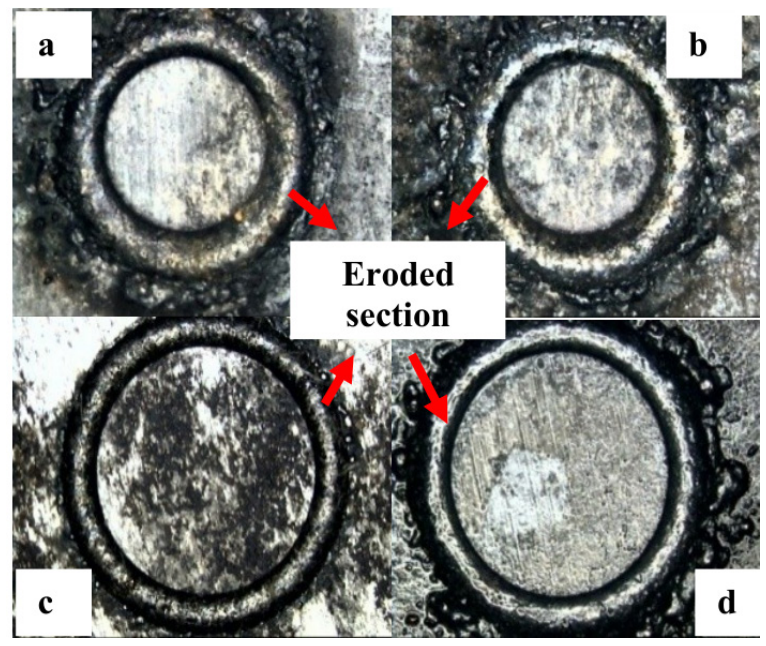

Fig. 5. EN -31 Sample machined by PMND-EDM; a) machined sample by tool 1, b) machined sample by tool 2, c) machined sample by tool 3, d) machined sample by tool 4

$\rightarrow 0 \%$ powder conc. $[g / 1]=2 \%$ powder conc. $[g / 1] \neq 4 \%$ powder conc. $[g / 1]$

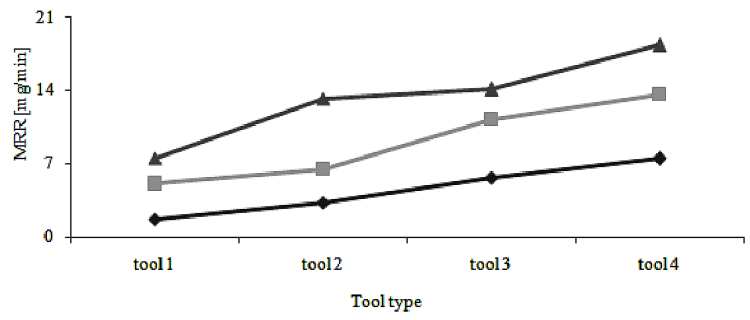

Fig. 6. Graph of MRR vs. tool type at different powder concentration

The plasma channel was small with a $2 \%$ concentration of powder due to which fewer craters can be seen after machining of the workpiece, as shown in Fig. 7. Intensive craters over the machined surface were observed in Fig. 8, which signifies that a higher erosion rate was achieved as the concentration of metallic powder was increased. A high energy enlarged plasma channel due to increased metallic powder concentration was the main reason for higher erosion. The electrical resistivity decreases, and the working gap expands due to the presence of metallic powder in EDM oil [3]. This phenomenon stabilizes the arc through better flushing and servo-hunting.

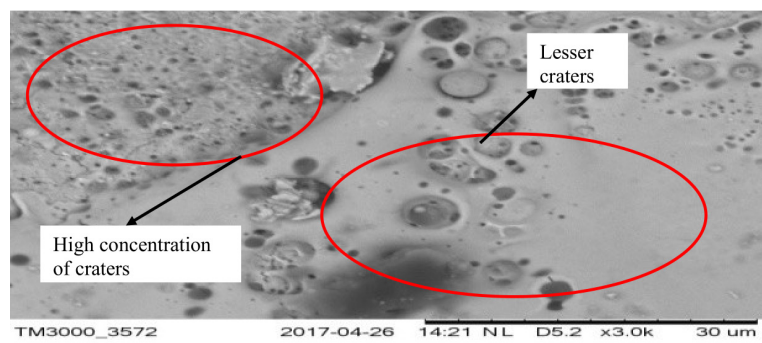

Fig. 7. SEM image of a machined surface with $2 \mathrm{~g} / \mathrm{I}$ powder concentration (image width: $68.8 \mu \mathrm{m}$, accelerating voltage $15.0 \mathrm{kV}$ )

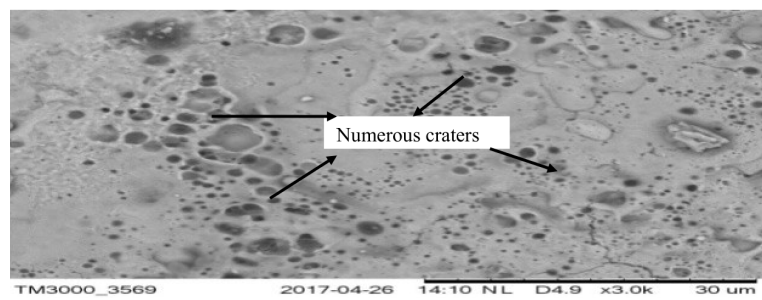

Fig. 8. SEM image of a machined surface with $4 \mathrm{~g} / \mathrm{l}$ powder concentration (image width: $68.8 \mu \mathrm{m}$, accelerating voltage $15.0 \mathrm{kV}$ )

\subsection{Influence of Machining Process on Surface Finish (Ra)}

Metallic powder additives along with dielectric oil reduce the electric density and increase the spark gap, due to which the sparking over the machined surface is uniformly distributed [23]. It was observed that higher surface finish was achieved in machined products because the increased spark gap helped in effective debris removal from the machining area [2]. Powder concentration and the type of metallic powder were important factors in influencing the spark gap. The maximum percentage increase in surface finish was $16.36 \%$ in PMND-EDM as compared to near-dry EDM as shown in Fig. 9. With a further increase of metallic powder concentration (4\%), the plasma channel becomes more uniformly energized and distributed. which gives better surface finish than that of the $2 \%$ powder concentration. 
Fig. 10 shows a machined surface with reduced cracks by PMND-EDM, which was also the reason to achieve better surface quality characteristics. Fig. 11 shows the surface of the workpiece machined at $4 \%$ powder concentration. The peaks signify the height of the surface irregularities at different parts over the machined area. The variation of surface roughness in the images was signified by the pattern of yellow colour. The dark yellow colour of the image shows the area of lesser irregularities while bright yellow colour signifies the area of high irregularities over the machined surface. Similar analysis was performed for other machined products with $2 \%$ powder concentration as well as in near-dry condition

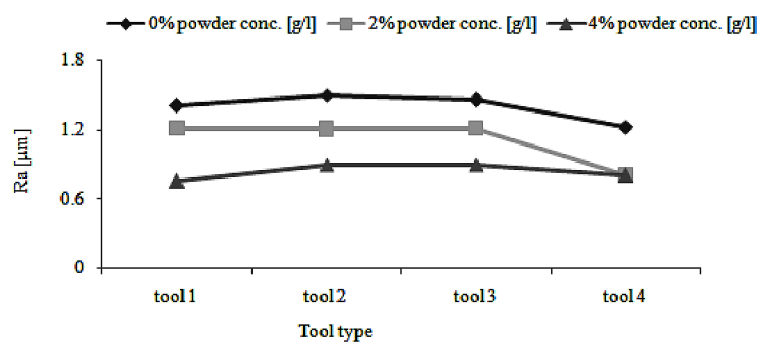

Fig. 9. Graph of surface roughness value vs. tool type at different powder concentration

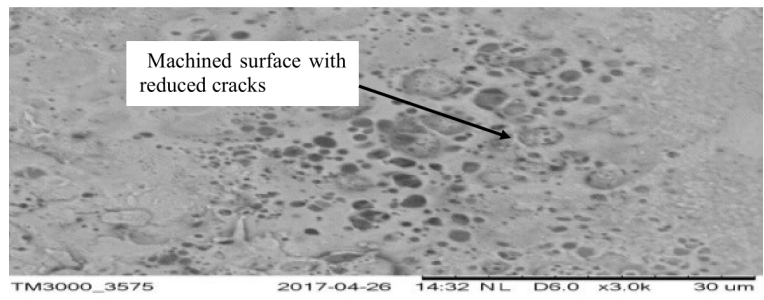

Fig. 10. SEM image of the machined area by PMND-EDM (Image width: $68.8 \mu \mathrm{m}$, accelerating voltage $15.0 \mathrm{kV}$ )

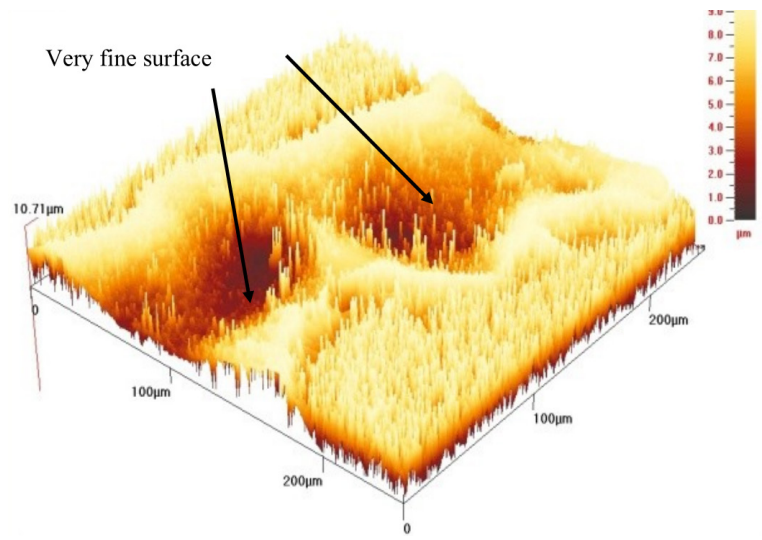

Fig. 11. 3-D image of surface roughness profile of the machined surface with $4 \mathrm{~g} / \mathrm{I} / \mathrm{I}$ powder in PMND-EDM as shown in Figs. 12 and 13, respectively. At $2 \%$ metallic powder concentration, the plasma is partially energized, which gives considerably better surface quality with lesser irregularities as shown in Fig. 12. Surface roughness decreases by adding zinc powder in the dielectric fluid, as added powder enlarges and widens the discharge gap between the electrodes. This results in the easy removal of debris, which leads to the improvement in surface quality. The powder particles distribute the spark energy uniformly, which results in shallow craters on the workpiece surface. It was revealed that by the addition of metallic powder, the discharging energy dispersion improves because insulating strength of the dielectric fluid reduces with the addition of metallic powder. These favourable conditions give a refined machined surface with a high surface finish. This proves that a significant role was played by the metallic powder to modify the channel

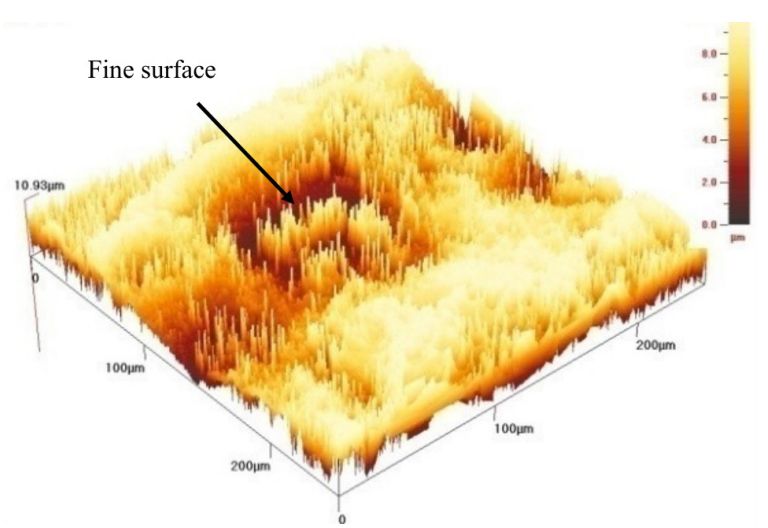

Fig. 12. 3-D image of surface roughness profile of the machined surface with $2 \mathrm{~g} / \mathrm{l} / \mathrm{I}$ powder in PMND-EDM

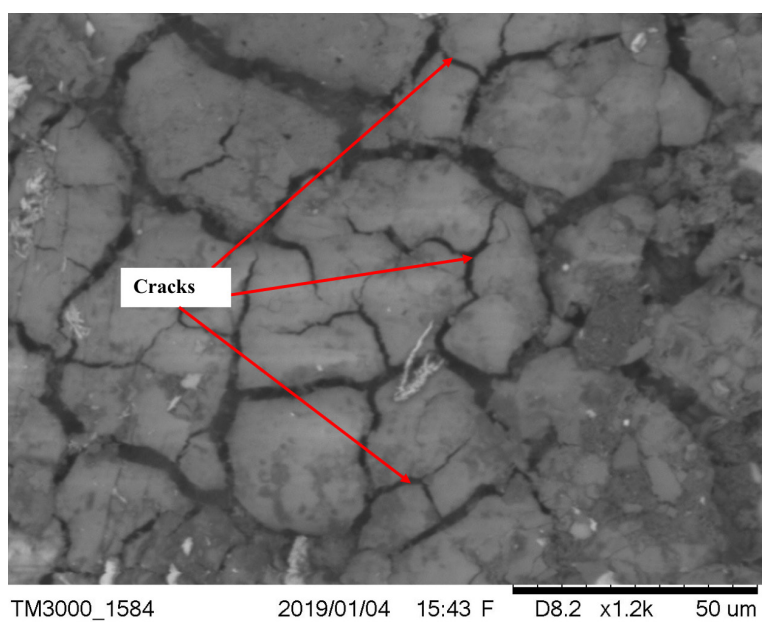

Fig. 13. SEM image of the machined area in near-dry EDM (image width: $68.8 \mu \mathrm{m}$, accelerating voltage $15.0 \mathrm{kV}$ ) 
of the plasma, which gives even and uniform surfaces. While in near-dry EDM, an improper plasma channel was observed due to the absence of metallic powder. This leads to the formation of a large machined area with an uneven surface with cracks, as shown in Figs. 13 and 14. The cross-sectional area was analysed for a

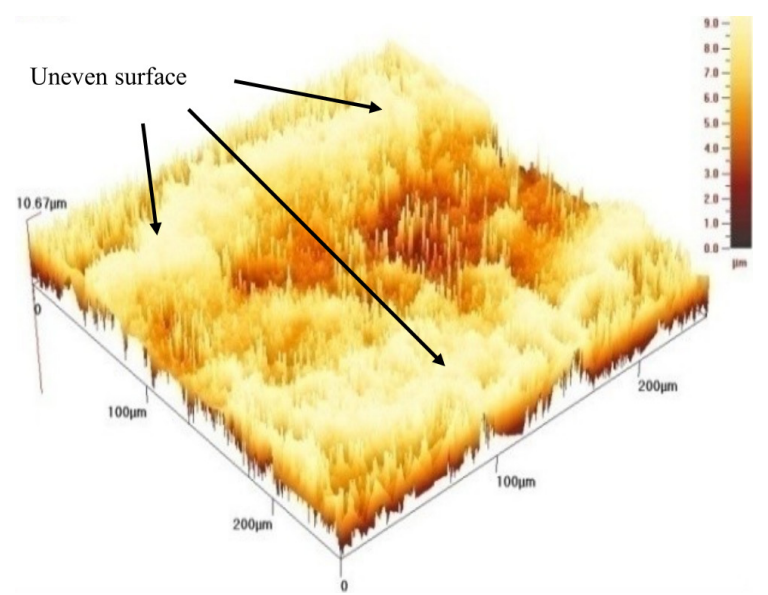

Fig. 14. 3-D image of surface roughness profile of machined surface with near-dry EDM

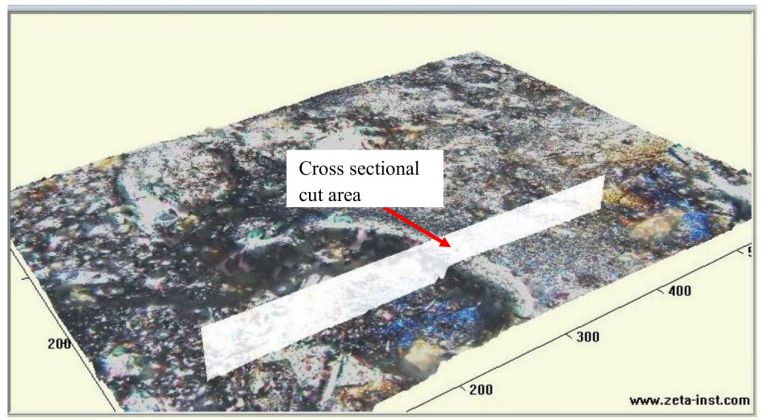

Fig. 15. 3-D image of the area for cross sectional study

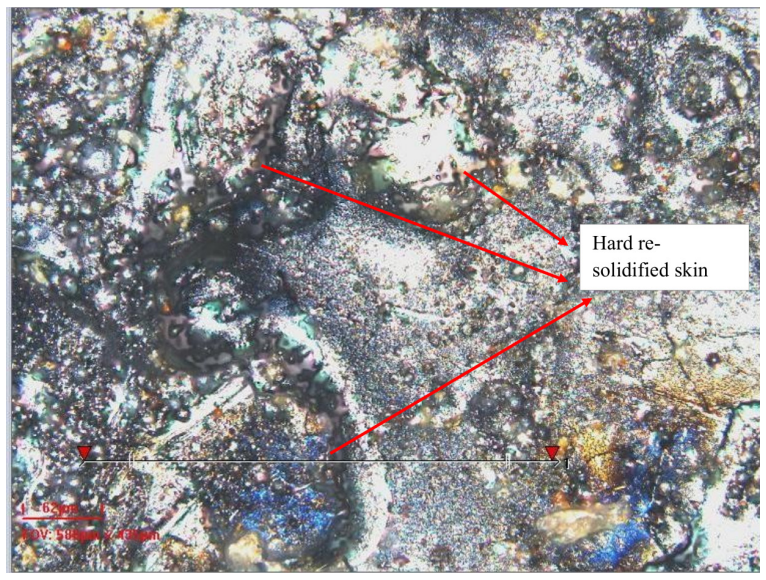

Fig. 16. Hard resolidified melted region at the cross-section of machined EN-31 sample re-melted zone at the machined area. The area cut for analysis is shown in Fig.15, while the cross-sectional re-solidified area of the molten metal is shown in Fig. 16.

\subsection{Analysis of Residual Stress of Machined Surfaces}

The residual stress analysis on the finished worksurfaces was performed with an X-ray residual analyzer (Pulstec limited model- $\mu$ X-360n). The machined workpiece sample information required for taking the measurement were lattice constant, crystal structure, diffraction plane, Young's modulus,
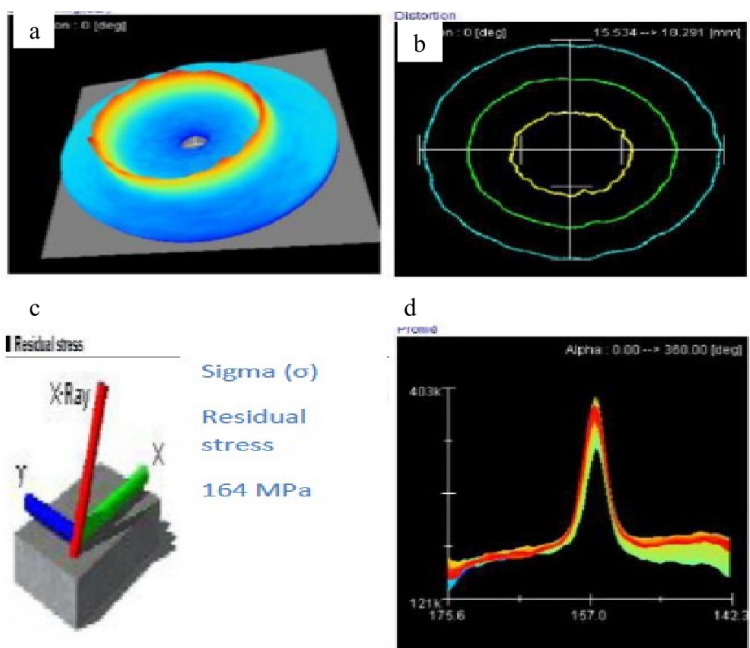

Fig. 17. Residual stress generated on work surface after machining without any metallic powder (ND-EDM); a) debye ring,

b) distortion ring, c) residual stress, and d) peak profile
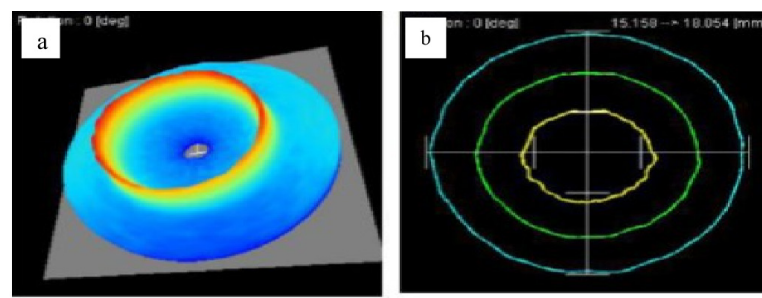$$
c
$$
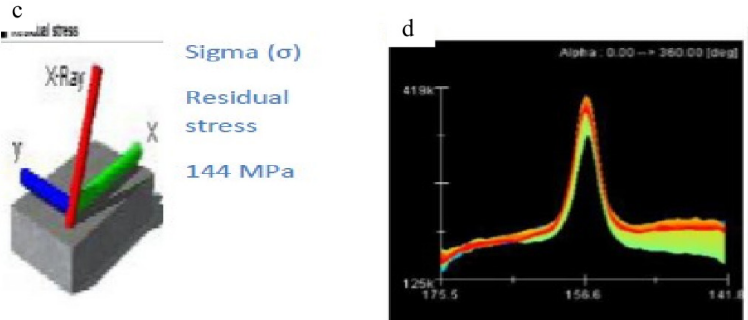

Fig. 18. Residual stress generated on work surface after machining with $2 \%$ metallic powder by PMND-EDM; a) debye ring, b) distortion ring, c) residual stress, and d) peak profile 
and Poisson ratio. The X-Ray residual stress measuring principle was based on Bragg's law. X-ray irradiation time was set up for 30 seconds while $\mathrm{X}$-ray tube current and voltage were $1 \mathrm{~mA}$ and 30 $\mathrm{kV}$, respectively. Sample distance was set at $39 \mathrm{~mm}$, and the angle of incidence of the X-ray was set at 35 degree while the wavelength of the X-ray was 2.29 A. All the measurements were taken at room temperature of $26{ }^{\circ} \mathrm{C}$. Fig. 17 shows the residual stress analysis of machined surface by ND-EDM at $0 \%$ powder concentration (ND-EDM). A Debye ring can be seen, which indicates residual stress distribution over the machined surface. The red ring signifies the region of maximum stress concentration while the yellow region signifies a region of comparatively lower stress induced at the machined surfaces, while the blue region signifies region of minimum induced stress. Other relevant information about the stress peak position and peak profile over the finished surface of the workpiece are also shown in Fig. 17. In ND-EDM, the residual stress measured by the machine was 164 $\mathrm{MPa}$ (tensile), as shown in Fig. 17. The value of stress of the machined surfaces by PMND-EDM with $2 \%$ metallic powder concentration was $144 \mathrm{MPa}$ (tensile), as shown in Fig. 18, which was lower compared to the stresses in the workpiece machined by ND-EDM. On further increasing the metallic powder concentration to $4 \%$ in PMND-EDM, the residual stresses further reduced to a value of $72 \mathrm{MPa}$ (tensile), as shown in Fig. 19. The comparative graph of different residual stresses of machined surfaces by ND-EDM and PMND-EDM is shown in Fig. 20. The maximum
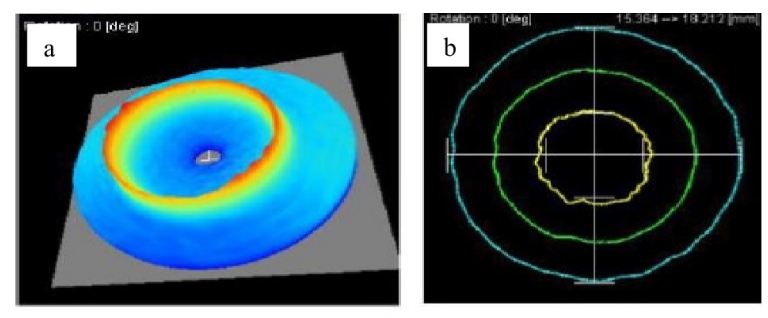

$$
\mathrm{c}
$$

Rasidal $\sin$
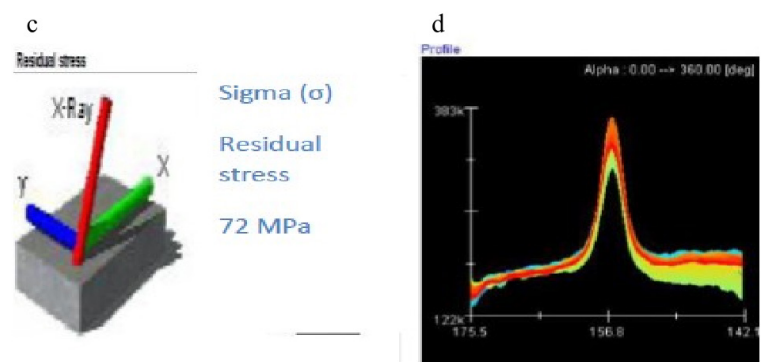

Fig. 19. Residual stress generated on work surface after machining with $4 \%$ metallic powder by PMND-EDM: a) debye ring, b) distortion ring, c) residual stress, and d) peak profile decrease in residual stress was found to be 56.09 $\%$ in PMND-EDM as compared to ND-EDM. The reason for the decrease in residual stresses in PMNDEDM can be attributed to the uniform distribution of heat or energy plasma over the machined surface and improved flushing condition, which ultimately relieves some part of the residual stresses over the machined surfaces.

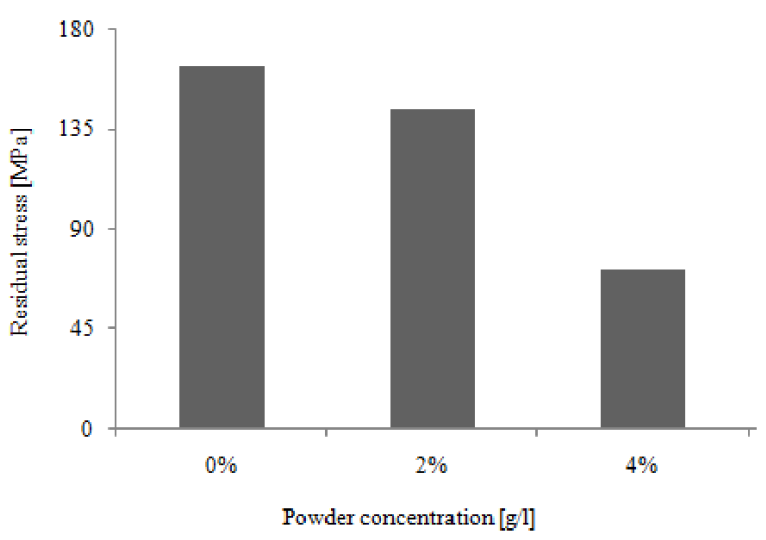

Fig. 20. Graph of the residual stress of machined products at different powder concentrations

\subsection{Analysis for Micro-Hardness of Machined Surface by PMND-EDM}

MH analysis for product machined by ND-EDM ana d PMND-EDM was performed with help of Fischerscope instrument (HM2000S model, USA). This instrument utilizes a carbide indenter which indents with respect to the increase in value of load applied. The topography of the machined surface of the workpiece was improved by metallic powder in the dielectric medium because these additives were responsible for reducing the surface pits over the machined surface. There was an improvement in surface properties with the addition of conductive powder in the dielectric medium due to the surface modification such as the formation of a solidified layer of zinc. The $\mathrm{MH}$ of the die steel workpiece was increased by adding chromium powder to the dielectric medium [24]. An $\mathrm{MH}$ of $1600 \mathrm{HV}$ was achieved with the addition of titanium powder to the dielectric [25]. This metallic powder leads to the formation of titanium carbide layer on carbon steel when machined with a copper tool.

In this study, the Vickers hardness number (HV) for machined part at $0 \%$ powder concentration was found to be $194 \mathrm{HV}$, but with powder additive machining this value increased to the maximum value 
of $520 \mathrm{HV}$ at $4 \%$ powder concentration. The powder additives changed the surface topography of the machined surface by forming a solidified recast layer, which was responsible for surface modification. This phenomenon increased the MH by $69.63 \%$, as shown in Fig. 21.

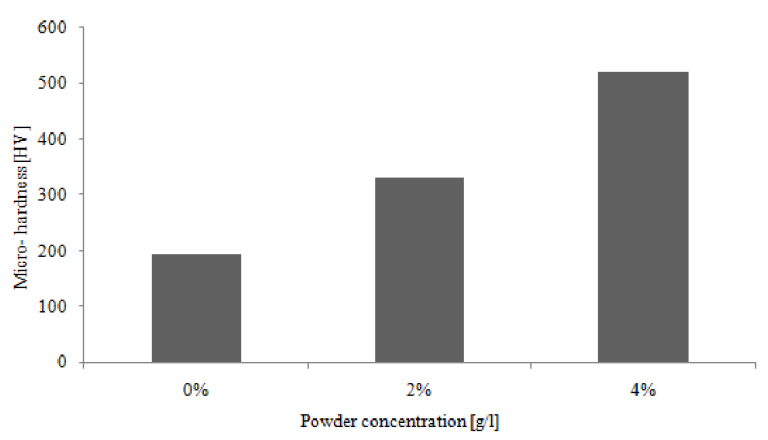

Fig. 21. $\mathrm{MH}$ of machined products at different metallic powder concentration

\section{CONCLUSIONS}

In this article, experimental investigations have been conducted regarding the PMND-EDM process. The metallic powder (zinc) as an additive along with a mist of dielectric medium was used for performance enhancements, and the following conclusions were made.

1. In PMND-EDM, the MRR was influenced by powder concentration. The MRR increases with an increase in metallic powder concentration and maximum increase in MRR was $17.85 \%$ as compared to ND-EDM.

2. Surface finish was improved by $16.36 \%$ in PMND-EDM as the sparking is uniformly distributed among the powder particles, which gives even and uniform machined surfaces.

3. There was a decrease in residual stress by 56.09 $\%$ over the machined surfaces in PMND-EDM. as compared to the ND-EDM process.

4. The maximum increase $\mathrm{MH}$ was $62.69 \%$ over the machined surface in PMND-EDM as compared to ND-EDM.

\section{REFRENCES}

[1] Tanimura, T., Isuzugawa, K., Fujita, I., Iwamoto, A., Kamitani, T. (1989). Development of EDM in the Mist. Proceedings of Ninth International Symposium of Electro Machining, vol. 9, p. 313316.

[2] Jeswani, M.L. (1981). Effects of the addition of graphite powder to kerosene used as the dielectric fluid in electrical discharge machining. Wear, vol. 70, no. 2, p. 133-139, DOI:10.1016/0043-1648(81)90148-4.

[3] Wong, Y.S., Lim, L.C., Rahuman, I., Tee, W.M. (1998).Nearmirror-finish phenomenon in EDM using powder-mixed dielectric. Journal of Materials Processing Technology, vol. 79, no. 1-3, p. 30-40, D0I:10.1016/S0924-0136(97)00450-0.

[4] Kansal, H.K., Singh, S., Kumar, P. (2005). Parametric optimization of powder mixed electric discharge machining by response surface methodology. Journal of Materials Processing Technology, vol. 169, no. 3, p. 427-436, DOI:10.1016/j.jmatprotec.2005.03.028.

[5] Tripathy, S., Tripathy, D.K. (2017). Optimization of process parameters and investigation on surface characteristics during EDM and powder mixed EDM. Bajpai, R., Chandrasekhar, U. (eds.). Innovative Design and Development Practices in Aerospace and Automotive Engineering. Lecture Notes in Mechanical Engineering. Springer, p. 385-391, DOI:10.1007/978-981-10-1771-1_41.

[6] Singh, J., Sharma, R.K. (2016). Assessing the effects of different dielectrics on environmentally conscious powdermixed EDM of difficult to machine material (WC-Co). Frontiers of Mechanical Engineering, vol.11, p. 374-387, D0l:10.1007/ s11465-016-0388-8.

[7] Bai, X., Zhang, Q.-H., Li, T.T., Zhang, Y.-H. (2012). Research on the medium breakdown mechanism of powder mixed near-dry electrical discharge machining. Chinese Journal of Mechanical Engineering, vol. 48, p. 186-192, D0I:10.3901/ JME.2012.07.186.

[8] Bai, X., Zhang, Q.H., Yang, T.Y., Zhang, J.H. (2013). Research on material removal rate of powder mixed neardry electrical discharge machining. International Journal of Advanced Manufacturing Technology, vol. 68, p. 1757-1766, DOl:10.1007/s00170-013-4973-2.

[9] Bai, X., Zhang, Q.H., Zhang, J.H., Kong, D., Yang, T. (2013). Machining efficiency of powder mixed near-dry electrical discharge machining based on different material combinations of tool electrode and workpiece electrode. Journal of Manufacturing Processes, vol. 15, no. 4, p.474-482, D0I:10.1016/J.jmapro.2013.09.005.

[10] Wang, X., Liu, Y., Zhang, Y., Sun, Q., Li, Z., Shen, Y. (2016). Characteristics of plasma channel in powder-mixed EDM based on monopulse discharge. International Journal of Advanced Manufacturing Technology, vol. 82, p. 1063-1069, D0l:10.1007/s00170-015-7236-6.

[11] Kumar, M., Datta, S., Kumar, R. (2018). Electro-discharge machining performance of Ti-6Al-4V Alloy: Studies on parametric effect and phenomenon of electrode wear. Arabian Journal for Science and Engineering, vol. 10, p. 1-16, DOI:10.1007/s13369-018-3632-1.

[12] Öpöz, T.T., Yasar, H., Ekmekci, N., Ekmekci, B. (2018). Particle migration and surface modification on $\mathrm{Ti}_{6} \mathrm{Al}_{4} \mathrm{~V}$ in $\mathrm{SiC}$ powder mixed electrical discharge machining. Journal of Manufacturing Processes, vol. 31, p. 744-758, D0l:10.1016/j. jmapro.2018.01.002.

[13] Khullar, V.R., Sharma, N. Kishore, S., Sharma, R. (2017). RSM- and NSGA-II-based multiple performance characteristicsoptimization of EDM Parameters for AISI 5160. 
Arabian Journal for Science and Engineering, vol. 42, p. 19171928, DOI:10.1007/s13369-016-2399-5.

[14] Liu, J.F., Guo, Y.B. (2016). Residual stress modeling in electric discharge machining (EDM) by incorporating massive random discharges. Procedia CIRP, vol. 45, p. 299-302, D0I:10.1016/j. procir.2016.02.060.

[15] Rao, P.S., Ramji, K., Satyanarayana, B. (2016). Effect of wire EDM conditions on generation of residual stresses in machining of aluminum 2014 T6 alloy. Alexandria Engineering Journal, vol. 55, no. 2, p. 1077-1084, D0l:10.1016/j. aej.2016.03.014.

[16] Shabgard, M., Seydi, S., Seyedzavvar, M. (2016). Novel approach towards finite element analysis of residual stresses in electrical discharge machining process. International Journal of Advanced Manufacturing Technology, vol. 82, p. 1805-1814, D0l:10.1007/s00170-015-7510-7.

[17] Kumar, S., Grover, S., Walia, R.S. (2018). Effect of hybrid wire EDM conditions on generation of residual stresses in machining of $\mathrm{HCHCr}$ D2 tool steel under ultrasonic vibration. International Journal on Interactive Design and Manufacturing, vol. 12, p. 11119-1137, D0l:10.1007/s12008018-0474-8.

[18] Gill, A.S., Kumar, S. (2018). Investigation of micro-hardness in electrical discharge alloyingof En31 tool steel with $\mathrm{Cu}-\mathrm{W}$ powder metallurgy electrode. Arabian Journal for Science and Engineering, vol.43, p. 1499-1510, D0I:10.1007/s13369-0172960-x.

[19] Chundru, V.R., Koona, R., Pujari, S.R. (2019). Surface modification of $\mathrm{Ti}_{6} \mathrm{Al}_{4} \mathrm{~V}$ alloy using EDMed electrode made with nano- and micron-sized TiC/Cu powder particles. Arabian
Journal for Science and Engineering, vol. 44, p. 1425-1436, DOI:10.1007/s13369-018-3561-z.

[20] Kumar, S., Batra, U. (2012).Surface modification of die steel materials by EDM method using Tungsten powder-mixed dielectric. Journal of Manufacturing Procesess, vol. 14, no. 1, p. 35-40, DOl:10.1016/j.jmapro.2011.09.002.

[21] Kumar, S., Singh, R., Batish, A., Singh, T.P., Singh, R. (2017). Investigating surface properties of cryogenically treated titanium alloys in powder mixed electric discharge machining. Journal of the Brazilian Society of Mechanical Sciences and Engineering, vol. 39, p. 2635-2648, D0l:10.1007/s40430016-0639-y.

[22] Sundriyal, S., Vipin, Walia, R.S. (2020). Experimental investigation on micro-hardness of EN-31 die steel in powder mixed near dry electric discharge machining method. Strojniški vestnik - Journal of Mechanical Engineering, vol. 66, no. 3, p. 184-192, D0l:10.5545/sv-jme.2019.6474.

[23] Zhao, W.S., Meng, Q.G., Wang, Z.L. (2002). The application of research on powder mixed EDM in rough machining. Journal of Materials Processing Technology, vol. 129, no. 1-3, p. 30-33, DOI:10.1016/S0924-0136(02)00570-8.

[24] Tripathy, S., Tripathy, D.K. (2017). An approach for increasing the micro-hardness in electrical discharge machining by adding conductive powder to the dielectric. Materials Today: Proceedings, vol. 4, no. 2, p. 1215-1224, D0l:10.1016/j. matpr.2017.01.140.

[25] Furutani, K., Saneto, A., Takezawa, H., Mohri, N., Miyake, H. (2001). Accertation of titanium carbide by electrical discharge machining with powder suspended in working fluid. Precision Engineering, vol. 25, no. 2, p. 138-144, D0I:10.1016/S0141$6359(00) 00068-4$. 